\title{
Crystal structure of Pseudomonas aeruginosa FabB C161A, a template for structure-based design for new antibiotics
}

\section{[version 1; peer review: 2 approved with reservations]}

\author{
Vladyslav Yadrykhins'ky (D), Charis Georgiou (D), Ruth Brenk (iD)
}

Department of Biomedicine, University of Bergen, Bergen, 5020, Norway

V1 First published: 01 Nov 2021, 10(Chem Inf Sci):1102

https://doi.org/10.12688/f1000research.74018.1

Latest published: 10 Jan 2022, 10(Chem Inf Sci):1102

https://doi.org/10.12688/f1000research.74018.2

\section{Abstract}

Background: FabB (3-oxoacyl-[acyl-carrier-protein] synthase 1) is part of the fatty acid synthesis II pathway found in bacteria and a potential target for antibiotics. The enzyme catalyses the Claisen condensation of malonyl-ACP (acyl carrier protein) with acyl-ACP via an acyl intermediate. Here, we report the crystal structure of the intermediate-mimicking Pseudomonas aeruginosa FabB (PaFabB) C161A variant.

Methods: His-tagged PaFabB C161A was expressed in E.coli Rosetta DE3 pLysS cells, cleaved by TEV protease and purified using affinity and size exclusion chromatography. Commercial screens were used to identify suitable crystallization conditions which were subsequently improved to obtain well diffracting crystals.

Results: We developed a robust and efficient system for recombinant expression of PaFabB C161A. Conditions to obtain well diffracting crystals were established. The crystal structure of PaFabB C161A was solved by molecular replacement at $1.3 \AA$ resolution.

Conclusions: The PaFabB C161A crystal structure can be used as a template to facilitate the design of FabB inhibitors.

Keywords crystal structure, 3-oxoacyl-[acyl-carrier-protein] synthase 1, FabB, antibiotics

Open Peer Review
Approval Status
version 2
(revision)
10 Jan 2022
01 Nov 2021
1. Bjarte A. Lund iD, UiT The Arctic University
of Norway, Tromsø, Norway
2. Klaus Reuter (iD), Philipps-Universität
Marburg, Marburg, Germany
Any reports and responses or comments on the
article can be found at the end of the article.

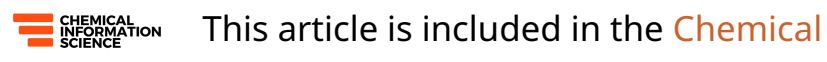

Information Science gateway. 


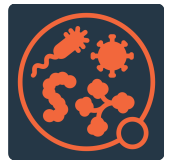

This article is included in the Pathogens

gateway.

Corresponding authors: Charis Georgiou (charis.Georgiou@uib.no), Ruth Brenk (ruth.brenk@uib.no)

Author roles: Yadrykhins'ky V: Formal Analysis, Investigation, Methodology, Visualization, Writing - Original Draft Preparation, Writing - Review \& Editing; Georgiou C: Conceptualization, Formal Analysis, Investigation, Methodology, Supervision, Visualization, Writing Original Draft Preparation, Writing - Review \& Editing; Brenk R: Conceptualization, Funding Acquisition, Project Administration, Supervision, Writing - Original Draft Preparation, Writing - Review \& Editing

Competing interests: No competing interests were disclosed.

Grant information: The work was supported by the Research Council of Norway (grant number 273588). We made use of the Facility for Biophysics, Structural Biology and Screening at the University of Bergen (BiSS), which has received funding from the Research Council of Norway (RCN) through the NORCRYST (grant number 245828) and NOR-OPENSCREEN (grant number 245922) consortia.

The funders had no role in study design, data collection and analysis, decision to publish, or preparation of the manuscript.

Copyright: @ 2021 Yadrykhins'ky V et al. This is an open access article distributed under the terms of the Creative Commons Attribution License, which permits unrestricted use, distribution, and reproduction in any medium, provided the original work is properly cited.

How to cite this article: Yadrykhins'ky V, Georgiou C and Brenk R. Crystal structure of Pseudomonas aeruginosa FabB C161A, a template for structure-based design for new antibiotics [version 1; peer review: 2 approved with reservations] F1000Research 2021, 10(Chem Inf Sci):1102 https://doi.org/10.12688/f1000research.74018.1

First published: 01 Nov 2021, 10(Chem Inf Sci):1102 https://doi.org/10.12688/f1000research.74018.1 


\section{Introduction}

New antibiotics are urgently needed to maintain the high standard of living that we have got accustomed to as the antibiotics of today are losing effectiveness faster than they are being replaced by new treatment options. ${ }^{1}$ If no action is taken, by 2050 drug-resistant infections will kill 10 million people a year worldwide, more than currently die from cancer. ${ }^{2}$ A possible source for new targets for antibiotics is the fatty acid synthesis (FAS II) pathway (Figure $1 \mathrm{~A}$ ). ${ }^{3}$ In this pathway, fatty acid synthesis is carried out by a series of monofunctional enzymes which are highly conserved among microbial pathogens. Genes coding for enzymes in the FAS II pathway have been found to be essential for $P$. aeruginosa in several genetic screens, including the gene for FabB (3-oxoacyl-[acyl-carrier-protein] synthase 1). ${ }^{4-8}$

Both, FabB and FabF (3-oxoacyl-[acyl-carrier-protein] synthase 2) catalyse the Claisen condensation of malonyl-ACP (acyl carrier protein) with acyl-ACP (Figure 1B), but differ in substrate specificity for the fatty acid chain. ${ }^{3}$ Platensimycin and platencin (Figure 1C) have been reported to be FabF and FabB inhibitors. ${ }^{9,10}$ However, it has been shown that these compounds do not bind potently to the w. t. enzyme, but only to the lauroyl-FabF intermediate (Figure 1B) and to intermediate-mimicking FabF variants in which the active site Cys has been changed to either Gln or Ala. ${ }^{9,11}$ It has been assumed that the same is the case for FabB.

To facilitate structure-based design of FAS II inhibitors, knowledge of the structures in this pathway is essential. Recently, we have reported the crystal structure of $P a \mathrm{FabF}$ and the reaction intermediate-mimicking variant $P a \mathrm{FabF}$ C164Q. ${ }^{12}$ Here, we report the crystal structure of $P a$ FabB C161A at $1.3 \AA$ resolution.

\section{Results}

Protein expression and purification

The gene coding for $P$. aeruginosa PA14 FabB C161A was synthesised and cloned in a bacterial plasmid pET-28a (+)-TEV vector after a DNA sequence coding for a 6-His-tag followed by a TEV cleavage site. To find good expression

(A)
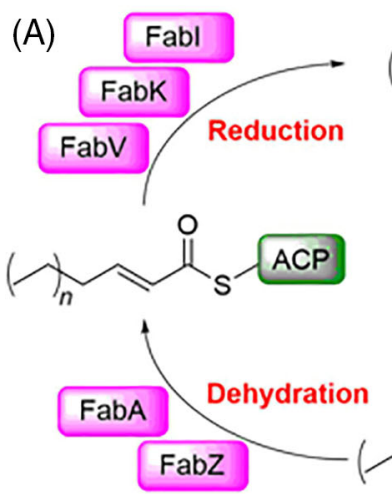

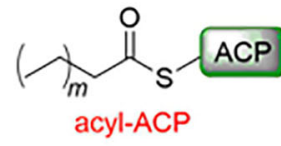

Chain increases with 2 C-atoms per cycle $(m=n+1)$
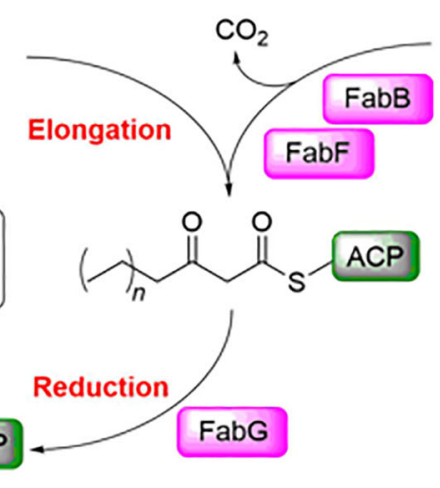<smiles>O=C([O-])CC(=O)SC1CC2C=CC21</smiles>

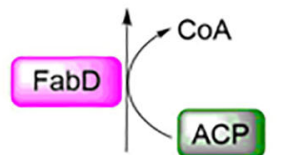

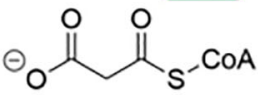

(B)

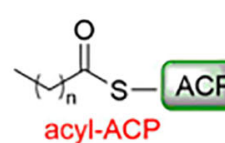<smiles>SCC[Pb]</smiles>

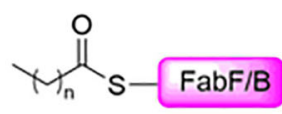

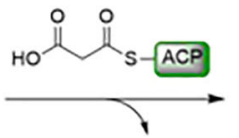<smiles>CCCCC(=O)CC(=O)SC1CCCC1</smiles>

$\mathrm{HS}-\mathrm{FabF/B}+\mathrm{CO}_{2}$

(C)

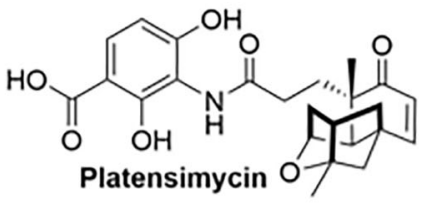<smiles>CC1(CCC(=O)Nc2c(O)ccc(C(=O)O)c2O)C2C=CC(CC2=O)C1C(=O)O</smiles>

Figure 1. FAS II pathway and its inhibitors. A) Schematic overview of the elongation part of the FAS II pathway. B) Condensation reaction catalysed by FabF/B. (ACP: acyl carrier protein). C) Platensimycin and platencin have been reported as dual FabF/B inhibitors. 
conditions, seven widely used $E$. coli strains were transformed with the plasmid (BL21 (DE3), BL21 (DE3) pLysS, C41 (DE3), C41 (DE3) pLysS, C43 (DE3), C43 (DE3) pLysS and Rosetta (DE3) pLysS) and screened for protein expression. The best results were obtained with Rosetta (DE3) pLysS cells (data not shown). Therefore, this cell line was used for all subsequent protein expression experiments. ${ }^{21}$

His-tagged $P a$ FabB C161A was purified using affinity chromatography with a Ni column followed by size exclusion chromatography (SEC). To obtain FabB with a cleaved His-tag, the protein obtained after affinity chromatography was cleaved with TEV protease. The cleaved protein was separated from the protease and the tag by inverse affinity chromatography followed by SEC. In both cases, pure protein was obtained as judged by SDS-PAGE gel electrophoresis (Figure 2). Typical yields for His-tagged $P a$ FabB C161A were $26 \mathrm{mg} / \mathrm{L}$ and for cleaved $P a$ FabB C161A $7 \mathrm{mg} / \mathrm{L}$.

\section{Crystallization of PaFabB C161A}

Crystallization trials of His-tagged $P a$ FabB C161A and cleaved $P a F a b B ~ C 164 A$ were attempted using the JCSG+, PACT premier, HELIX (only His-tagged $P a F a b B$ C161A) and LFS screens. No promising crystallization conditions for Histagged $\mathrm{PaFabB} \mathrm{C} 161 \mathrm{~A}$ were found using these screens. In contrast, 11 different conditions resulted in crystals of His-tag cleaved $P a$ FabB C164A (Table 1, Figure 2). All of these conditions contained PEG 3350 between 20 and 25\% and a number of conditions contained ethylene glycol. Further, the majority of the conditions contained $0.1 \mathrm{M}$ Bis-Tris propane, and 0.2 M sodium iodide. Therefore, these components were kept for further optimization trials. The $\mathrm{pH}$ of the initial conditions varied from 5.5 to 8.5. As crystals grown in a buffer of $\mathrm{pH} 7.5$ were visually judged to be more regular (e. g. the crystal shown in Figure 3B), this $\mathrm{pH}$ was fixed during optimization. These considerations resulted in an optimization matrix where the concentration of PEG 3350 was varied between 5 and $30 \%$ and the protein concentration between 9 and $23 \mathrm{mg} / \mathrm{mL}$. Ethylene glycol was added to all conditions at either 10 or $20 \%$ while $0.2 \mathrm{M}$ sodium iodide and

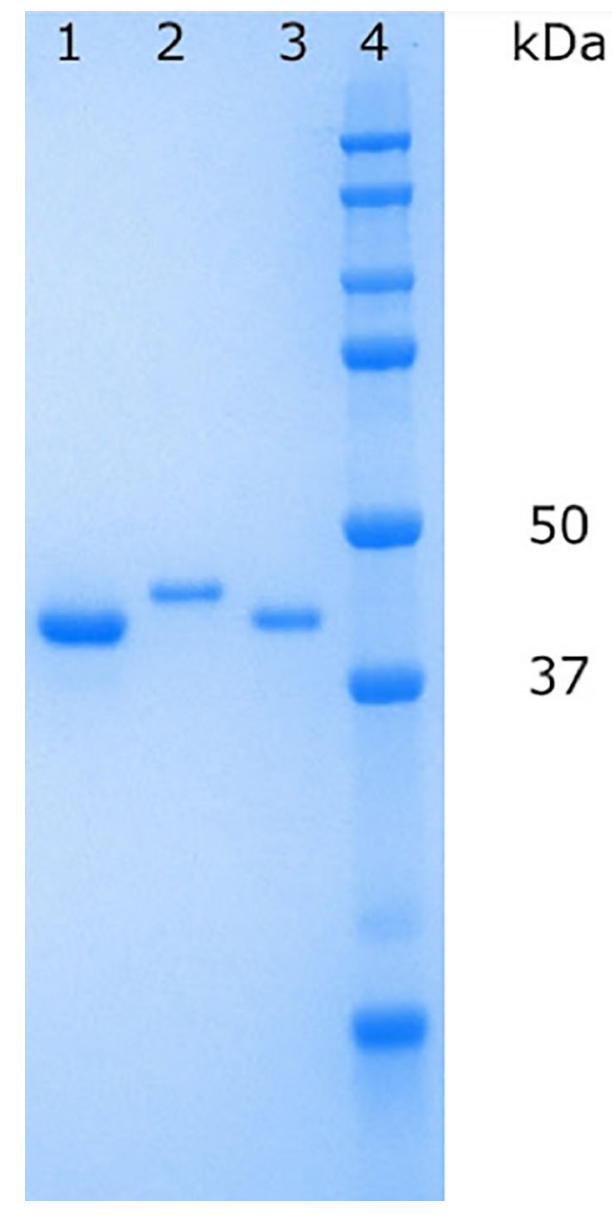

Figure 2. Protein samples on an SDS-PAGE gel. Lane 1: PaFabB C164A (without His-tag) after inverse affinity chromatography, lane 2: 6-His-tagged PaFabB C164A after SEC purification, lane 3: PaFabB C164A (without His-tag) after SEC purification, lane 4: protein ladder. 
Table 1. Conditions in which crystals of PaFabB C161A were formed. (PEG-polyethylene glycol; EG-ethylene glycol.)

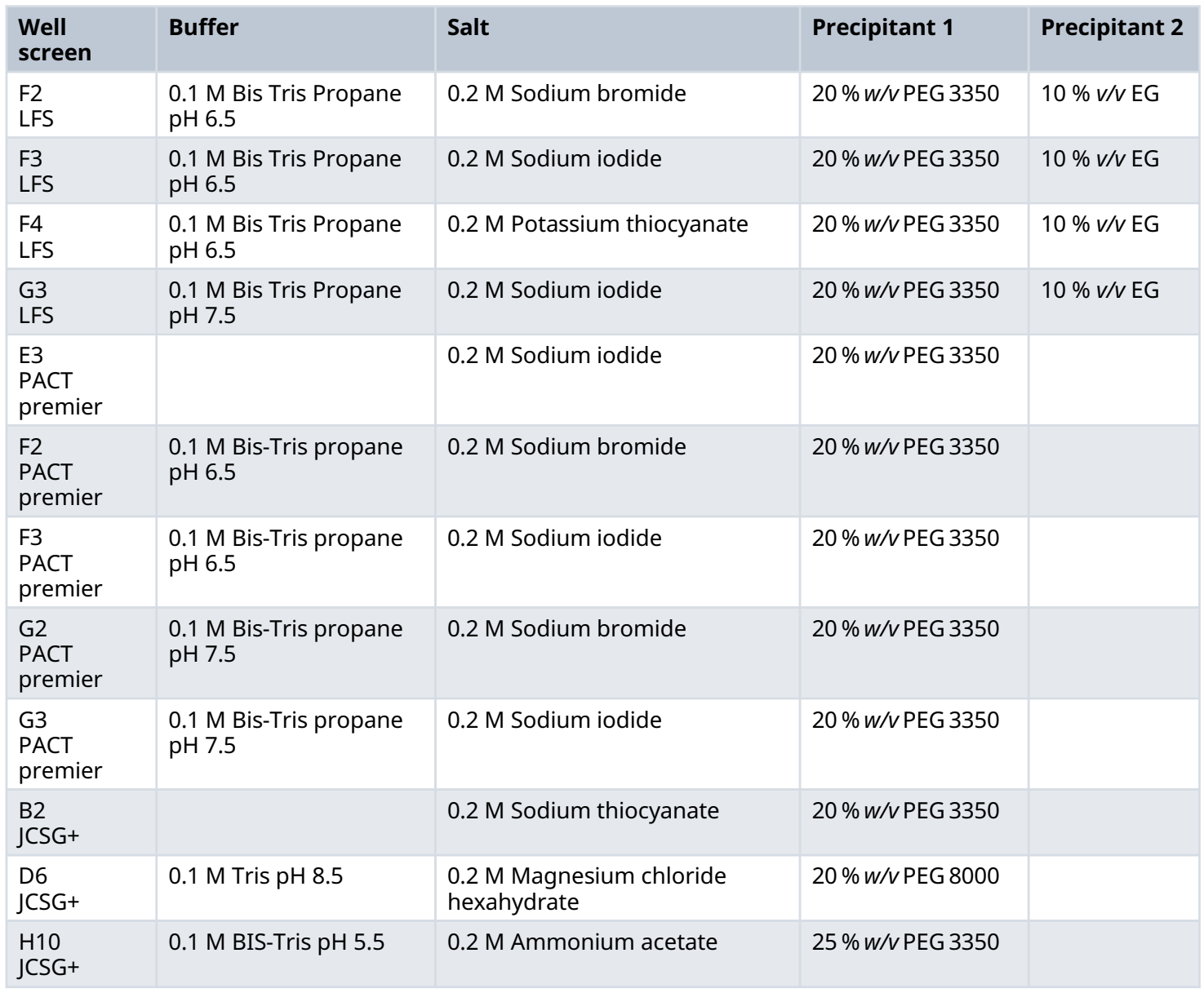

0.1 M Bis-Tris propane were fixed (Figure 4). Under 32 conditions, crystals were obtained. These were mounted and used for diffraction experiments.

Six different conditions led to well-diffracting crystals (Figure 4). For these, data sets with resolutions between 2 and $1.3 \AA$ could be collected. For the best diffracting crystal, the crystal structure was determined using a homology model created based on Vibrio cholerae FabB (VcFabB PDB Id 4XOX) as search model (Table 2). The crystal was in the space group C 2221 and contained 2 protein molecules in the asymmetric unit.

\section{Crystal structure of PaFabB C161A}

$P a F a b B$ C161A has the same overall fold as observed before for FabB and FabF from other organisms (Figure 5 and Figure 6A). The rmsd between $P a \mathrm{FabB} \mathrm{C} 161 \mathrm{~A}$ and $V c \mathrm{FabB}$ (the protein with the highest sequence identity in the PDB (72\%), PDB Id 4XOX) is $0.424 \AA$ while the rmsd to the w.t. PaFabF (sequence identity 41\%, PDB Id 4JPF) is $0.843 \AA$. The two catalytic histidines, His 296 and His 331, are highly conserved and well aligned with the catalytic histidines from both $V c$ FabB as well as the ones from $P a$ FabF (Figure 6B).

Due to the high concentration of ethylene glycol $(20 \% \mathrm{v} / \mathrm{v})$ and salt in the well and protein buffers, respectively $(150 \mathrm{mM}$ $\mathrm{NaCl}$ and $200 \mathrm{mM} \mathrm{NaI}), 18$ ethylene glycol molecules and 11 ions $\left(\mathrm{Cl}^{-}\right.$and $\left.\mathrm{I}^{-}\right)$were identified and placed in the crystal structure of $\mathrm{PaFabB} \mathrm{C} 161 \mathrm{~A}$ during refinement (Figure 7A). Some of these molecules were found to bind in the active site of the protein (Figure 7B). The chloride ion $\mathrm{Cl} 1$ binds tightly (B factor for $\mathrm{Cl} 1$ is $18 \AA^{2}$, average $\mathrm{B}$-factor for protein atoms is $16.8 \AA^{2}$, average ions $\mathrm{B}$ factors is $30.7 \AA^{2}$ ) in the active site of chain $\mathrm{B}$, in close proximity to the catalytic residues His 296 (3.3 $\AA$ ) and His 331 (3.2 $\AA$ ). Moreover, $\mathrm{Cl} 1$ forms two additional interactions with an ethylene glycol (EDO511, average $\mathrm{B}$ factor $18 \AA^{2}$ ) and a water molecule $(\mathrm{HOH} 227)$ in the active site. 


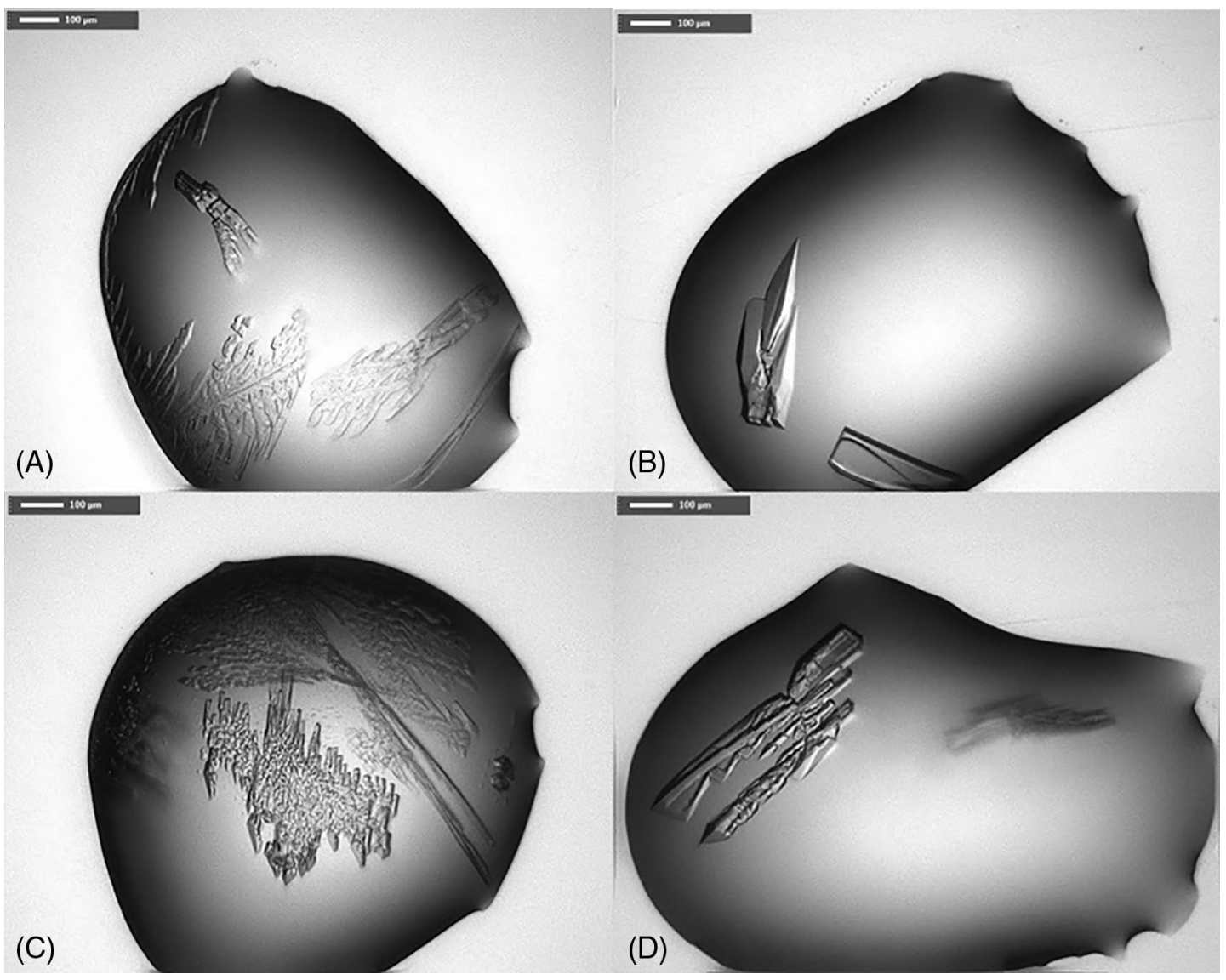

Figure 3. Selected crystals obtained from various screens. A) condition F3 from LFS, B) G3 from LFS, C) F3 from PACT premier, D) G3 from PACT premier (for composition of crystallization buffer see Table 1).

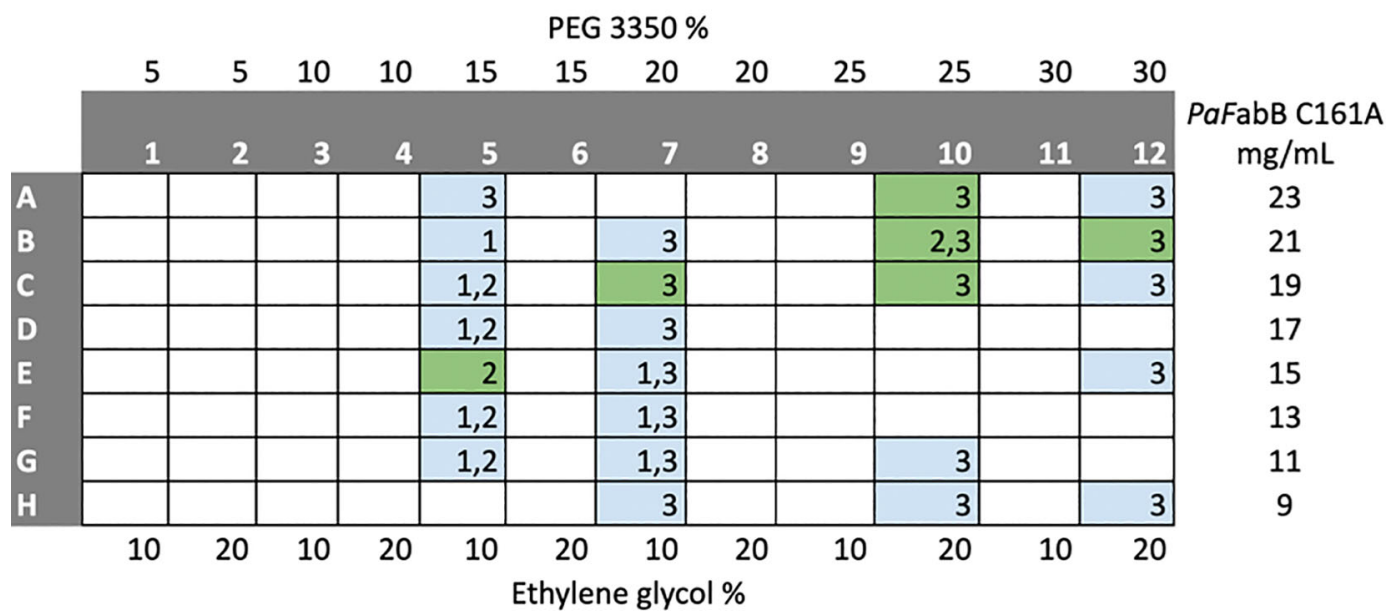

Figure 4. Plate layout for optimization of crystallization conditions. The numbers in the cells indicate the ratio between protein solution and crystallization buffer in the drops (drop 1-1:1 ratio, drop 2-1:2 ratio, drop 3-2:1 ratio). Coloured cells indicate conditions from which crystal were harvested and mounted for diffraction experiments. Green cells indicate conditions under which diffracting crystals were obtained.

\section{Active site and differences to PaFabF}

Phe 400/391 (numbering based on $P a \mathrm{FabF} / \mathrm{PaFabB}$ ) was previously identified, as one of the highly conserved active site residues, to play a pivotal role in substrate specificity and ligand binding, by adopting a different conformation between 
Table 2. Data-collection and refinement statistics of PaFabB C161A. Values in parentheses are for the highest resolution shell.

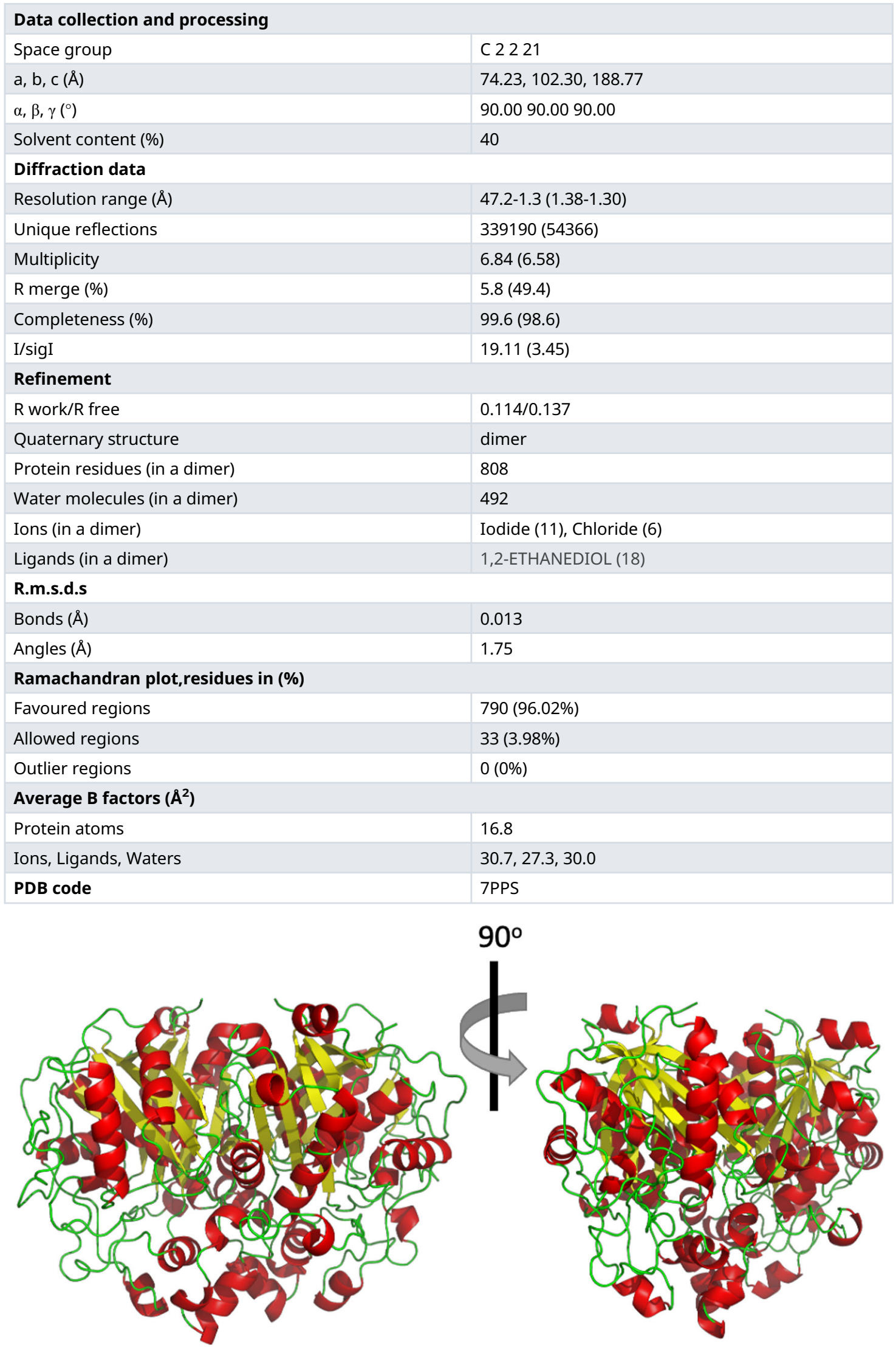

Figure 5. Cartoon representation showing the tertiary structure of PaFabB C161A. Beta sheets and alpha helices are shown in yellow and red colour respectively while loops are shown in green. 


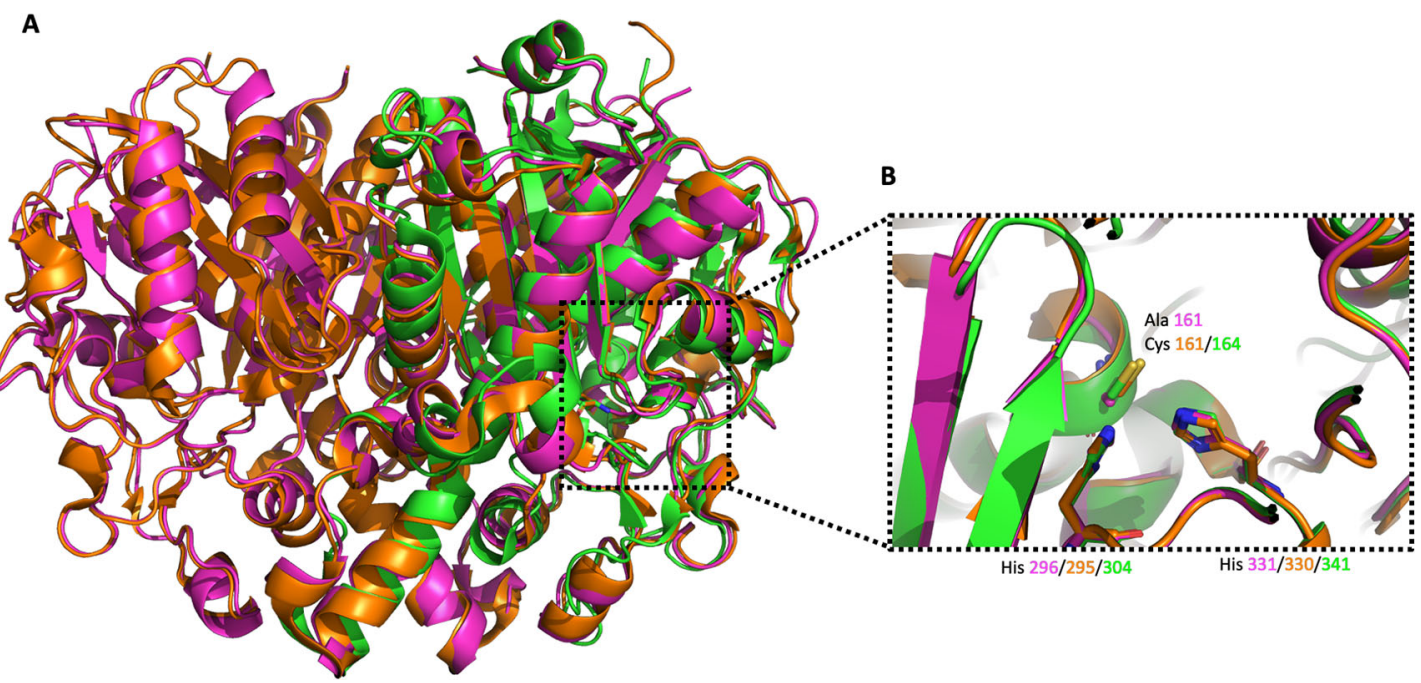

Figure 6. Alignment of VcFabB (PDB Id 4XOX), PaFabF (PDB Id 4JPF) and PaFabB C161A. A) The three different enzymes are shown in orange, green and magenta cartoon style, respectively. B) Alignment of the active site catalytic triad of VcFabB, w. t. PaFabF and PaFabB C161A.

A B

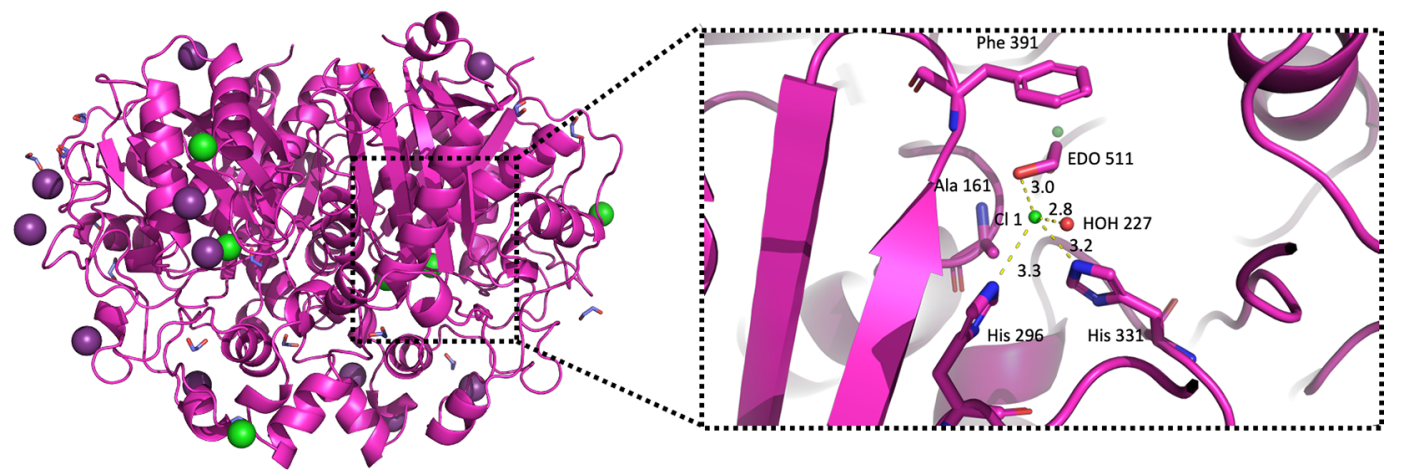

Figure 7. Crystal structure of $P a F a b B$ C161A. A) The structure of $P a F a b B$ is shown as magenta cartoon style. Iodine and chloride ions are shown with deep purple and green colour respectively. B) Active site residues are shown as magenta sticks, water molecules and chloride ions are shown as red and green spheres, respectively, while the distances between the chloride ion $\mathrm{Cl} 1$ and the neighbouring molecules are shown as yellow dashed lines.

the w.t. and the intermediate state of the FabF enzyme. ${ }^{9,12}$ In w. t. $P a$ FabF (PDB Id 4JPF, Figure $8 \mathrm{~A}$ and B), Phe400 is in a 'closed' conformation (dihedral angle C-CA-CB-CG $=-177.1^{\circ}$ ). When mutating the catalytic residue Cys 164 to Gln 164 (PDB Id 7OC1 - Figure 8A and C) the enzyme has been shown to mimic the intermediate state and to trap the Phe 391 into the 'open' conformation (dihedral angle C-CA-CB-CG $=168.8^{\circ}$ ). Here, the catalytic residue Cys 161 of $P a$ FabB was mutated to Ala 161. As can be seen from the crystal structure (Figure 8A and D), Phe 391 adopts the 'open' conformation as expected for an intermediate-mimicking FabB variant (dihedral angle $\mathrm{C}-\mathrm{CA}-\mathrm{CB}-\mathrm{CG}=170.2^{\circ}$ ). Although, the overall sequence identity between $P a \mathrm{FabB}$ and $P a \mathrm{FabF}$ is only $41 \%$, the conservation in the active site is much higher (Figure 9). Apart from Thr 271 in FabF that is mutated to Val 268 in FabB, all active site residues involved in hydrogen-bond interactions with platensimycin are conserved between the two enzymes (Figure 8A). That makes it highly likely that ligands binding into the active site of FabF may also bind to FabB with a similar affinity, and thus opens up the possibility for the designing of dual inhibitors for both FabF and FabB that will lead to a complete inhibition of the last step of the fatty acid elongation cycle.

\section{Discussion}

In this study, the first high-resolution crystal structure of $P a F a b B ~ C 161 \mathrm{~A}$ is reported. This structure can now serve as a template for the structure-based design of FabB inhibitors. The C161A mutation of FabB in this crystal structure causes Phe 391 to be in the 'open' conformation (Figure 8) and allows targeting of the intermediate-acylated state of FabB; in a 
A

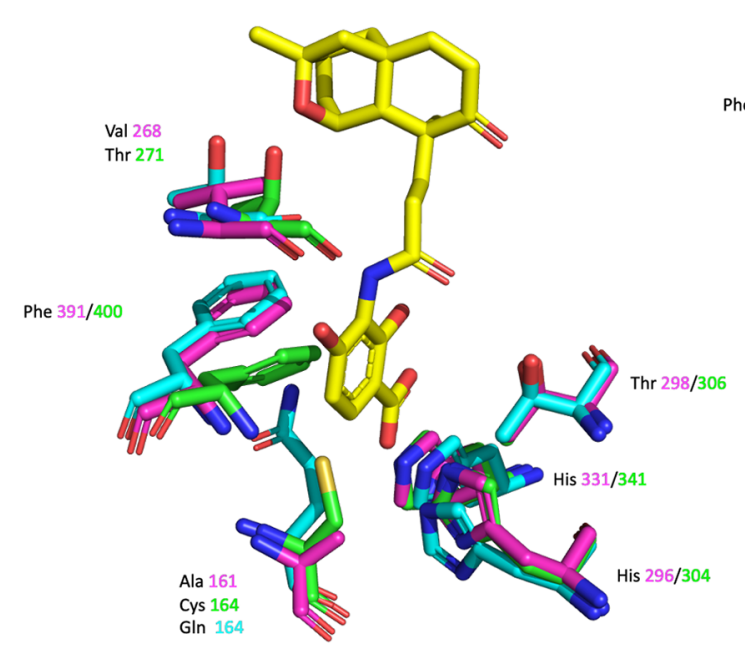

B

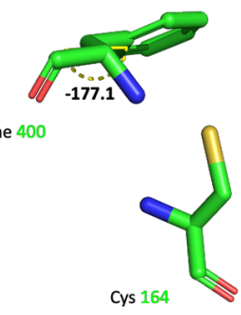

C

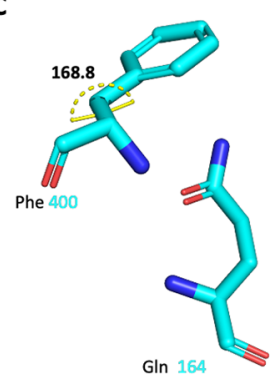

D
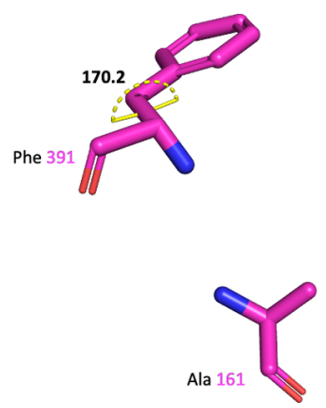

Figure 8. Alignment of PaFabF (PDB Id 4JPF), PaFabF C164Q (PDB Id 70C1) and PaFabB C161A. A) The active site residues of the three different enzymes are shown as green, cyan and magenta sticks, respectively. Platensimycin binding to PaFabF C164Q (PDB Id 7OC1) is shown as yellow sticks. Side chain conformation and dihedral angle C-CACB-CG of Phe391/400 is shown in B) for PaFabF C) for PaFabF (164Q and D) for PaFabB C161A.

A

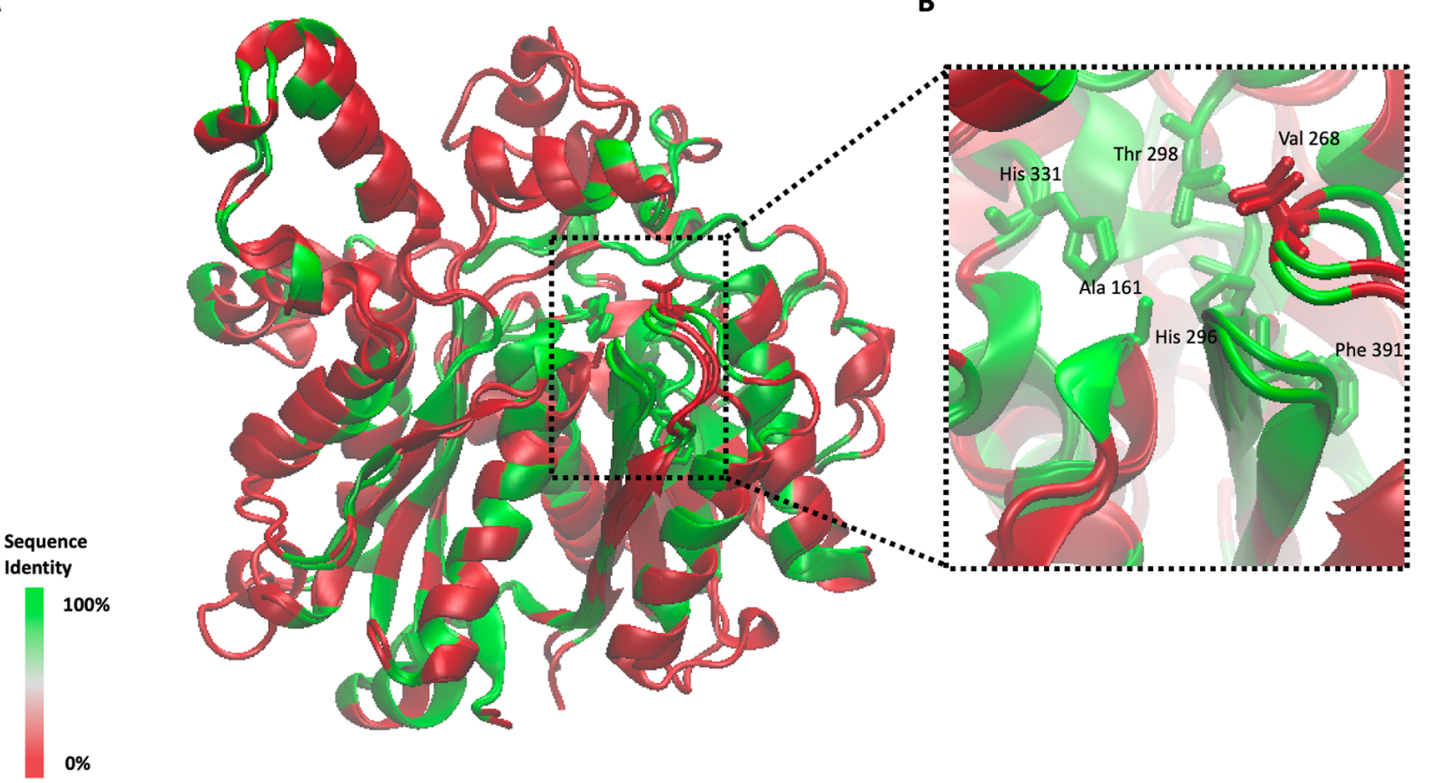

Figure 9. Sequence identity conservation between PaFabB C161A and PaFabF (PDB Id 4JPF). A) Alignment between chain A of $P a F a b F$ and $P a F a b B ~ C 161 A$ is shown as cartoon style and color-coded based on the sequence identity between the two isoenzymes. B) Zoom in showing the active site and active site residues. Residue labels and numbering are based on PaFabB only.

similar manner to the natural antibiotic platensimycin. Furthermore, due to the high conservation of the overall fold and the high sequence identity in the active site between the structure reported here with PaFabF C164Q (PDB Id 7OC1), both structures can be used as a template for the design of novel dual FabF/B inhibitors. 


\section{Methods}

Recombinant protein production and purification

The gene coding for $P$. aeruginosa PA14 FabB (ORF number (open reading frame): PA14_43690), with a single point mutation C161A was synthesised and cloned in a bacterial plasmid pET-28a(+)-TEV vector using the cloning sites NdeI/ BamHI by Genscript. The plasmid had a DNA sequence coding for a 6-His-tag followed by a TEV cleavage site before PaFabB. Seven different E. coli strains (OverExpress C41(DE3) SOLOs and C43(DE3) SOLOs from Biosearch technologies; BL-21(DE3), BL-21(DE3) pLysS, C41(DE3) pLysS and C43(DE3) pLysS from Lucigen, and Rosetta (DE3) pLysS from Merck) were heat-shock transformed with the synthesised plasmid. Expression of $P a$ FabB in each transformed cell line was tested as per manufacturer protocol.

E.coli Rosetta (DE3) pLysS competent cells yielded the highest protein expression, based on SDS-PAGE analysis, and were used as an expression system for large-scale protein production and purification. Transformed cells were inoculated in $50 \mathrm{~mL}$ of LB medium supplemented with kanamycin $(30 \mu \mathrm{g} / \mathrm{mL})$ and chloramphenicol $(50 \mu \mathrm{g} / \mathrm{mL})$ overnight at $310 \mathrm{~K}$. Pre-culture stocks were prepared by mixing the overnight culture with glycerol (final concentration $40 \% \mathrm{v} / \mathrm{v}$ ), aliquoted and kept in $-80^{\circ} \mathrm{C}$ until use. For large-scale expression, $0.1 \mathrm{~mL}$ of pre-culture stock was inoculated in $100 \mathrm{~mL}$ of LB medium supplemented with kanamycin $(30 \mu \mathrm{g} / \mathrm{mL})$ and chloramphenicol $(50 \mu \mathrm{g} / \mathrm{mL})$ overnight at $310 \mathrm{~K}$. The entire volume was then transferred into $900 \mathrm{~mL}$ of LB-medium containing antibiotics and the cell growth continued until $\mathrm{OD}_{600}$ reached 0.7 . Protein expression was then induced by adding IPTG to a final concentration of $1 \mathrm{mM}$ and the expression continued for another 3-3.5 hours.

Cells were harvested by centrifugation $(15$ minutes, $5000 \mathrm{~g}, 277 \mathrm{~K})$, resuspended in lysis buffer $(20 \mathrm{mM} \mathrm{Tris-HCl}$, $500 \mathrm{mM} \mathrm{NaCl}, 20 \mathrm{mM}$ imidazole, $1 \mathrm{mM}$ DTT, $10 \%$ glycerol $(v / v)$, pH 7.4) with addition of one tablet of Complete EDTAfree protease inhibitor cocktail (Roche) and incubated with magnet stirring for 60 minutes at $277 \mathrm{~K}$. $20 \mathrm{U}$ (units) of DNAse I (Sigma Aldrich) was added per cell pellet, before the mixture was sonicated on ice by an ultrasonic processor (Sonics, Vibra-Cell VC130) for a total of two minutes with 10 seconds pulses with amplitude 70 . The debris and insoluble protein were pelleted by centrifugation at $15000 \mathrm{rpm}, 277 \mathrm{~K}$, for 30 minutes. The supernatant was collected and filtered with Whatman filter units $0.2 \mu \mathrm{M}$ (GE healthcare) using a syringe. The protein was then purified using a $\mathrm{Ni}^{2+} \mathrm{Sepharose}^{2}$ High Performance HisTrap HP $5 \mathrm{~mL}$ column (GE Healthcare) with an increasing imidazole gradient from 0 to $500 \mathrm{mM}$. The fractions containing $\mathrm{PaFabB}$ C161A were pooled and TEV protease was added to remove the affinity tag. The

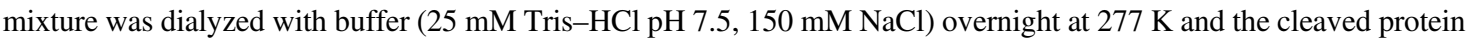
was purified by passage through a $\mathrm{Ni}^{2+}$ HisTrap column. SEC was then performed on a HiLoad 26/600 Superdex $75 \mathrm{pg}$ column (Cytiva) with equilibration buffer (20 mM Tris-HCl, $150 \mathrm{mM} \mathrm{NaCl}, 1 \mathrm{mM} \mathrm{DTT}$, pH 7.4). Purity was confirmed by SDS-PAGE (Mini-PROTEAN TGX Stain-Free Precast Gel; Bio-Rad) and the final concentration of $P a$ FabB C161A was determined using a NanoDrop ND-1000 (Thermo Fisher Scientific).

\section{Crystallization and X-ray data collection}

For crystallization trials JCSG+ (MD1-37), PACT premier (MD1-29) and LFS (Ligand Friendly Screen, MD1-122) crystallization screens from Molecular Dimensions were used. His-tag cleaved $P a$ FabB C161A $(23 \mathrm{mg} / \mathrm{mL})$ in $20 \mathrm{mM}$ Tris- $\mathrm{HCl}, 150 \mathrm{mM} \mathrm{NaCl}, 1 \mathrm{mM}$ DTT, $\mathrm{pH}$ 7.4, was mixed with well buffer in different ratios $(2: 1,1: 1$ and 1:2) on a Triple Sitting Drop 96-well plate (TTP Labtech) using a crystallography Mosquito LCP (TTP LabTech). The plates were incubated at $20^{\circ} \mathrm{C}$. Optimization (Figure 4 ) of the initial hit conditions (Table 2) was achieved by varying the precipitants and protein concentrations while keeping the salt and buffer concentration constant. Optimisation led to rod-shaped crystals $(250 \times 100 \times 10 \mu \mathrm{m})$ in multiple drops (Figure 3$)$.

Crystals with a final concentration of precipitant lower than $25 \%(\mathrm{w} / \mathrm{v})$ were cryoprotected with a mixture consisting of the crystallization buffer and Cryomix 9 from CryoSol MD1-90 (Molecular Dimensions) (final composition of the cryomixture: $0.2 \mathrm{M}$ NaI, 0.1 M Bis-Tris propane $\mathrm{pH} 7.5,5 \%(w / v)$ PEG 3350, $10 \%(v / v)$ EG $5 \%(v / v)$, diethylene glycol, $5 \%(v / v)$ 1,2-propanediol, $5 \%(v / v)$ dimethyl sulfoxide, $5 \%(v / v)$ glycerol, 5 mM NDSB 201 (3-(1-Pyridinio)-1propanesulfonate), $5 \%(\mathrm{v} / \mathrm{v})$ 1,4-dioxane) prior to flash-cooling in liquid nitrogen.

X-ray data were collected from single crystals at the DESY synchrotron (Hamburg, Germany) at the P11 high-throughput MX beamline. In each case, crystals were maintained at $100 \mathrm{~K}$ and the X-ray wavelength was $0.976246 \AA$. Data were processed with the automatic data processing pipeline of P11 beamline, using XDS. ${ }^{13}$

\section{Structure solution and refinement}

The structure was solved by molecular replacement using Dimple ${ }^{14}$ from the CCP4i2 suite. ${ }^{15}$ As search model, a homology model generated from wt. $V c F a b B$ (PDB Id 4XOX) with $72 \%$ sequence identity was used. Refinement was performed using REFMAC $5{ }^{16}$ while inspection of electron-density and difference density maps and model manipulation 
was achieved using Coot $^{17}$ During refinement, water molecules, ions and side-chain conformers were included. The model geometry was assessed using MolProbity, ${ }^{18}$ the PDB redo server ${ }^{19}$ and the PDB validation tools. The crystallographic data and refinement statistics are listed in Table 2. The figures were generated with PyMOL v.2.4.1 (Schrödinger, LLC) and VMD v.1.9.3. ${ }^{20}$

\section{Data availability}

Protein Data Bank: The crystal structure of PaFabB C161A with the PDB Id 7PPS, https://doi.org/10.2210/pdb7PPS/ $\mathrm{pdb}^{21}$

\section{Acknowledgements}

We acknowledge DESY (Hamburg, Germany), a member of the Helmholtz Association HGF, for the provision of experimental facilities. Parts of this research were carried out at PETRA III and we would like to thank Johanna Hakanpää and Sofiane Saouane for assistance in using the P11 beamline. Beamtime was allocated for the proposal BAG-20190768 EC. We thank Khan Kim Dao for excellent support with protein purification and Ludvik Olai Espeland for help with preparing Figure 1.

1. Brown ED, Wright GD: Antibacterial Drug Discovery in the Resistance Era. Nature. 2016; 529(7586): 336-343. PubMed Abstract | Publisher Full Text

2. O'Neill J: Review on Antimicrobial Resistance. Tackling DrugResistant Infections Globally: Final Report and Recommendations. 2016 Reference Source.

3. Yao J, Rock CO: Bacterial Fatty Acid Metabolism in Modern Antibiotic Discovery. Biochimica et Biophysica Acta (BBA) - Molecular and Cell Biology of Lipids. 2017; 1862(11): 1300-1309. PubMed Abstract | Publisher Full Text | Free Full Text

4. Poulsen $B E$, Yang R, Clatworthy $A E$, et al.: Defining the Core Essential Genome of Pseudomonas Aeruginosa. PNAS. 2019; 116(20): 10072-10080.

PubMed Abstract | Publisher Full Text | Free Full Text

5. Turner KH, Wessel AK, Palmer GC, et al.: Essential Genome of Pseudomonas Aeruginosa in Cystic Fibrosis Sputum. PNAS. 2015; 112(13): 4110-4115

PubMed Abstract | Publisher Full Text | Free Full Text

6. Lee SA, Gallagher LA, Thongdee M, et al.: General and ConditionSpecific Essential Functions of Pseudomonas Aeruginosa. PNAS. 2015; 112(16): 5189-5194.

PubMed Abstract | Publisher Full Text | Free Full Text

7. Skurnik D, Roux D, Aschard H, et al.: A Comprehensive Analysis of in vitro and in vivo Genetic Fitness of Pseudomonas Aeruginosa Using High-Throughput Sequencing of Transposon Libraries. PLoS Pathog. 2013; 9(9): e1003582.

PubMed Abstract | Publisher Full Text | Free Full Text

8. Liberati NT, Urbach JM, Miyata S, et al.: An Ordered, Nonredundant Library of Pseudomonas Aeruginosa Strain PA14 Transposon Insertion Mutants. PNAS. 2006; 103(8): 2833-2838. PubMed Abstract | Publisher Full Text | Free Full Text

9. Wang J, Soisson SM, Young K, et al.: Platensimycin Is a Selective FabF Inhibitor with Potent Antibiotic Properties. Nature. 2006; 441(7091): 358-361.

PubMed Abstract | Publisher Full Text

10. Wang J, Kodali S, Lee SH, et al.: Discovery of Platencin, a Dual FabF and FabH Inhibitor with in vivo Antibiotic Properties. PNAS. 2007; 104(18): 7612-7616.

PubMed Abstract | Publisher Full Text | Free Full Text

11. Singh $\mathrm{SB}$, Ondeyka JG, Herath $\mathrm{KB}$, et al.: Isolation, Enzyme-Bound Structure and Antibacterial Activity of Platencin A1 from
Streptomyces Platensis. Bioorg. Med. Chem. Lett. 2009; 19(16): 4756-4759.

PubMed Abstract | Publisher Full Text

12. Baum B, Lecker LSM, Zoltner M, et al.: Structures of Pseudomonas Aeruginosa $\beta$-Ketoacyl-(Acyl-Carrier-Protein) Synthase II (FabF) and a C164Q Mutant Provide Templates for Antibacterial Drug Discovery. Acta Crystallogr. F: Struct. Biol. Commun. 2015; 71(8): 1020-1026.

PubMed Abstract | Publisher Full Text | Free Full Text

13. Kabsch W: XDS. Acta Crystallogr. D Biol. Crystallogr. 2010; 66(Pt 2): 125-132.

PubMed Abstract | Publisher Full Text | Free Full Text

14. Wojdyr M, Keegan R, Winter G, et al.: DIMPLE - a Pipeline for the Rapid Generation of Difference Maps from Protein Crystals with Putatively Bound Ligands. Acta Crystallogr. A. 2013; 69(a1): s299. Publisher Full Text

15. Potterton L, Agirre J, Ballard C, et al.: CCP4i2: The New Graphical User Interface to the CCP4 Program Suite. Acta Crystallogr D Struct Biol. 2018; 74(Pt 2): 68-84.

PubMed Abstract | Publisher Full Text | Free Full Text

16. Murshudov GN, Skubák P, Lebedev AA, et al.: REFMAC5 for the Refinement of Macromolecular Crystal Structures. Acta Cryst $D$. 2011; 67(4): 355-367.

PubMed Abstract | Publisher Full Text | Free Full Text

17. Emsley $\mathrm{P}$, Cowtan K: Coot: Model-Building Tools for Molecular Graphics. Acta Crystallogr. D Biol. Crystallogr. 2004; 60(Pt 12 Pt 1): 2126-2132. Publisher Full Text

18. Williams CJ, Headd JJ, Moriarty NW, et al.: MolProbity: More and Better Reference Data for Improved All-Atom Structure Validation. Protein Sci. 2018; 27(1): 293-315. PubMed Abstract | Publisher Full Text | Free Full Text

19. Joosten RP, Long F, Murshudov GN, et al.: The PDB_REDo Server for Macromolecular Structure Model Optimization. IUCrJ. 2014; 1(Pt 4): 213-220.

PubMed Abstract | Publisher Full Text | Free Full Text

20. Humphrey W, Dalke A, Schulten K: VMD: Visual Molecular Dynamics. J. Mol. Graph. 1996; 14(1): 33-38. PubMed Abstract | Publisher Full Text

21. Georgiou C, Brenk R, Yadrykhinsky V: Announcing the worldwide Protein Data Bank. Nat. Struct. Biol. 2003; 10: 980. Publisher Full Text 


\section{Open Peer Review}

\section{Current Peer Review Status: ? ?}

\section{Version 1}

Reviewer Report 06 December 2021

https://doi.org/10.5256/f1000research.77724.r101462

(C) 2021 Reuter K. This is an open access peer review report distributed under the terms of the Creative Commons Attribution License, which permits unrestricted use, distribution, and reproduction in any medium, provided the original work is properly cited.

\section{Klaus Reuter}

Institut für Pharmazeutische Chemie, Philipps-Universität Marburg, Marburg, Germany

First of all: Apart from the fact that I do not insist on CC* or CC1/2 values in Table 2, I do absolutely agree with every single concern of the $1^{\text {st }}$ reviewer making it unnecessary to restate all these points.

\section{Further (minor) issues:}

Page 3, Introduction, $1^{\text {st }}$ paragraph, line 3: Replace "drug-resistant infections" with "infections caused by drug-resistant pathogens".

Page 3, Introduction, $2^{\text {nd }}$ paragraph, line 4: Replace "w.t. enzyme" with "apo-enzyme".

Page 3, Figure 1 (B): In the first partial reaction of the FabF/B-catalysed reaction, the authors indicate the incoming HS-FabF/B (above the reaction arrow) but forget to indicate the leaving HS-ACP.

Page 4, Results/Protein expression and purification, $2^{\text {nd }}$ paragraph, line 2: Replace “... FabB with a cleaved His-tag ..." with“... FabB lacking the His-tag ...".

Page 4, Results/Crystallization of PaFabB C161A, $1^{\text {st }}$ paragraph, line 2: Replace "cleaved FabB" with "FabB lacking the His-tag".

Page 5, Results/Crystal structure of PaFabB C161A, $1^{\text {st }}$ paragraph, line 3: rmsd values for superimposed structures are given with three significant digits, respectively, which suggests inappropriately high accuracy. $0.424 \AA$ should be replaced by $0.42 \AA$ and $0.843 \AA$ by $0.84 \AA$.

Page 5, Results/Crystal structure of PaFabB C161A, $1^{\text {st }}$ paragraph, line 5: Replace “... from both VcFabB as well as the ones from PaFabB ..." with"... from both VcFabB and PaFabB ...".

Page 6, Results/Active site and differences to $P a F a b F, 1^{\text {st }}$ paragraph, line 1 : The $1^{\text {st }}$ sentence 
of this paragraph has to be reformulated. It does not make sense in its present form.

Page 7, Table 2: Again, several values are given with inappropriately high accuracy. Multiplicity: 6.84 (6.56) should be replaced by 6.8 (6.6); I/sig(I): 19.11 (3.45) should be replaced by 19.1 (3.5); Ramachandran plot, residues in (\%): (96.02\%) should be replaced by $(96.0 \%)$ and $(3.98 \%)$ by $(4.0 \%)$.

Page 8, Results/Active site and differences to PaFabF, $1^{\text {st }}$ paragraph, line 1: Replace "w.t." with "apo-".

Page 8, Results/Active site and differences to $P a F a b F, 1^{\text {st }}$ paragraph, line 2: Replace "When mutating ..." with "Upon the mutation of ...".

Page 8, Results/Active site and differences to PaFabF, $1^{\text {st }}$ paragraph, line 8: Replace “... is mutated to Val268 ..." with "... is replaced by Val268 ..." or "... corresponds to Val268 ...".

Figure 5: It is not immediately obvious if the figure shows the homodimer or a single subunit only. This information should be given. If the complete dimer is shown (which I presume) it would make sense to present its subunits in different colours or shades of colour. The same applies to subsequent figures.

\section{Major concern:}

The many figures showing FabB or the superpositions of different FabB/F enzymes are not very meaningful. FabB and FabF differ in substrate specificity for the fatty acid chain. The authors should definitely discuss the reasons for the respective substrate specificity by means of available structures.

I wish the authors had explained why the $\mathrm{C} 161 \mathrm{~A}$ mutation leads to the open conformation of the active site (see reviewer 1 ).

In summary, the manuscript is scientifically sound and significant, although somewhat greater effort should have been put into its presentation.

Is the work clearly and accurately presented and does it cite the current literature? Partly

Is the study design appropriate and is the work technically sound? Yes

Are sufficient details of methods and analysis provided to allow replication by others? Yes

If applicable, is the statistical analysis and its interpretation appropriate? Not applicable

Are all the source data underlying the results available to ensure full reproducibility? Yes 


\section{Are the conclusions drawn adequately supported by the results?}

Yes

Competing Interests: No competing interests were disclosed.

Reviewer Expertise: protein crystallography; enzymology

I confirm that I have read this submission and believe that I have an appropriate level of expertise to confirm that it is of an acceptable scientific standard, however I have significant reservations, as outlined above.

Author Response 22 Dec 2021

\section{Ruth Brenk}

Thank you Klaus for reviewing our paper and for very constructive feedback!

Below follows a point-to-point reply to all issues that you have raised.

First of all: Apart from the fact that I do not insist on CC* or CC1/2 values in Table 2, I do absolutely agree with every single concern of the $1^{\text {st }}$ reviewer making it unnecessary to restate all these points.

We have addressed all points that Bjarte Lund has raised. Please refer to the reply to his comments to see what we have changed.

Page 3, Introduction, $1^{\text {st }}$ paragraph, line 3: Replace "drug-resistant infections" with "infections caused by drug-resistant pathogens".

Done

Page 3, Introduction, $2^{\text {nd }}$ paragraph, line 4: Replace "w.t. enzyme" with "apo-enzyme".

Done

Page 3, Figure 1 (B): In the first partial reaction of the FabF/B-catalysed reaction, the authors indicate the incoming HS-FabF/B (above the reaction arrow) but forget to indicate the leaving HS-ACP.

Corrected as suggested

Page 4, Results/Protein expression and purification, $2^{\text {nd }}$ paragraph, line 2: Replace “... FabB with a cleaved His-tag ..." with"... FabB lacking the His-tag ...".

Done

Page 4, Results/Crystallization of PaFabB C161A, $1^{\text {st }}$ paragraph, line 2: Replace "cleaved FabB" with "FabB lacking the His-tag".

Done

Page 5, Results/Crystal structure of PaFabB C161A, $1^{\text {st }}$ paragraph, line 3: rmsd values for superimposed structures are given with three significant digits, respectively, which suggests inappropriately high accuracy. $0.424 \AA$ should be replaced by $0.42 \AA$ and $0.843 \AA$ by $0.84 \AA$.

Done

Page 5, Results/Crystal structure of PaFabB C161A, $1^{\text {st }}$ paragraph, line 5: Replace “... 
from both VcFabB as well as the ones from PaFabB ..." with"... from both VcFabB and PaFabB ...".

Done

Page 6, Results/Active site and differences to PaFabF, $1^{\text {st }}$ paragraph, line 1: The $1^{\text {st }}$ sentence of this paragraph has to be reformulated. It does not make sense in its present form.

This sentence reads now: "The highly conserved Phe400/391 (numbering based on PaFabF/PaFabB) in the malonyl-CoA binding site was previously identified to play a pivotal role in substrate specificity and ligand binding, as this residue adopts different conformations in the apo and the intermediate-binding state."

Page 7, Table 2: Again, several values are given with inappropriately high accuracy. Multiplicity: 6.84 (6.56) should be replaced by 6.8 (6.6); I/sig(I): 19.11 (3.45) should be replaced by 19.1 (3.5); Ramachandran plot, residues in (\%): (96.02\%) should be replaced by $(96.0 \%)$ and $(3.98 \%)$ by $(4.0 \%)$.

Done

Page 8, Results/Active site and differences to PaFabF, $1^{\text {st }}$ paragraph, line 1: Replace "w.t." with "apo-".

Done

Page 8, Results/Active site and differences to PaFabF, $1^{\text {st }}$ paragraph, line 2: Replace "When mutating ..." with "Upon the mutation of ...".

Done

Page 8, Results/Active site and differences to $P a F a b F, 1^{\text {st }}$ paragraph, line 8: Replace “... is mutated to Val268 ..." with "... is replaced by Val268 ..." or "... corresponds to Val268 ...".

Done

Figure 5: It is not immediately obvious if the figure shows the homodimer or a single subunit only. This information should be given. If the complete dimer is shown (which I presume) it would make sense to present its subunits in different colours or shades of colour. The same applies to subsequent figures.

We have added the information to the relevant figures (now 6 and 7) and colored the subunits as suggested.

The many figures showing FabB or the superpositions of different FabB/F enzymes are not very meaningful. FabB and FabF differ in substrate specificity for the fatty acid chain. The authors should definitely discuss the reasons for the respective substrate specificity by means of available structures.

We have inserted a new section to discuss the substrate selectivity with respect to fatty acids "Comparison of fatty acid chain binding channel in and in PaFabB and PaFabF". In addition, we have deleted the old Figure 5.

I wish the authors had explained why the C161A mutation leads to the open conformation of the active site (see reviewer 1 ).

An explanation has been added to what is now the second paragraph in the section "Comparison of malonyl-CoA binding sites in PaFabB and PaFabF".

We hope that we have answered all issues that you have raised to your full satisfaction. If not, please let us know what else we should modify. 
Competing Interests: No competing interests were disclosed.

Reviewer Report 15 November 2021

https://doi.org/10.5256/f1000research.77724.r98455

(C) 2021 Lund B. This is an open access peer review report distributed under the terms of the Creative Commons Attribution License, which permits unrestricted use, distribution, and reproduction in any medium, provided the original work is properly cited.

\section{Bjarte A. Lund}

Hylleraas Centre for Quantum Molecular Sciences, Department of Chemistry, UiT The Arctic University of Norway, Tromsø, Norway

Yadrykhins'ky et al. present an interesting story on the exploration of a new system for the development of new antibiotics. The authors make a convincing case for why fatty acid synthesis is a good target. Furthermore, the authors convey a clear technical step towards the goal by engineering a mutant variant of one enzyme in the pathway which mimics the intermediate step of the enzymatic reaction. An intermediate state that has been found to be susceptible to other inhibitors.

My objections to this paper are not on the technical and scientific soundness of the work but on its presentation.

The introduction is short and does not clearly convey to me as a non-expert how the mutation of a Cys to GIn/Ala would make the enzyme resemble the intermediate state. I need to read all the way until Figure 9 and the Discussion to be informed about the Pheresidue that flips between open/closed conformations.

After reading the paper, it is still not clear to me whether GIn was tried, or whether other amino acids could have had the same effect. Perhaps it is not important since Ala apparently worked.

In the Abstract, acyl-intermediate could more clearly be written as acyl-enzyme intermediate.

Results, Protein expression, and purification, TEV site would more precisely be termed TEV protease cleavage site.

Results, Crystallization (Figure 2) C164A/C161A is mixed non-consistently. I can only assume this is a mix-up with the group's other work with FabF. But it is very confusing to the reader.

Clearly out of scope to the paper, but it would have been interesting to know if iodinephasing was tested. 
It is unclear how the resolution cutoff was decided. I/sig(I) is still very high at the highest resolution shell. It would also have been useful if CC* or CC1/2 was presented in Table 2 .

The very short Discussion-segment could have been merged with the Results-section. The discussion does raise the very interesting possibility of dual-target inhibitors that would presumably be of high interest with regards to the emergence of resistance. This could have been emphasized earlier as well.

Methods, The sonication amplitude appears to lack a unit, presumably \%. For the protein concentration determination, the extinction coefficient used should be given.

Reference 21 appears to be a mistake.

All in all, I did appreciate the work and look forward to the continuation of the story.

Is the work clearly and accurately presented and does it cite the current literature? Partly

Is the study design appropriate and is the work technically sound?

Yes

Are sufficient details of methods and analysis provided to allow replication by others? Yes

If applicable, is the statistical analysis and its interpretation appropriate?

Not applicable

Are all the source data underlying the results available to ensure full reproducibility? Yes

Are the conclusions drawn adequately supported by the results?

Yes

Competing Interests: No competing interests were disclosed.

Reviewer Expertise: Biochemistry, structural biology, enzymology,

I confirm that I have read this submission and believe that I have an appropriate level of expertise to confirm that it is of an acceptable scientific standard, however I have significant reservations, as outlined above.

Author Response 22 Dec 2021

Ruth Brenk

Thank you Bjarte for reviewing our article! 
Here follows a point-to-point reply to the issues that you have raised.

The introduction is short and does not clearly convey to me as a non-expert how the mutation of a Cys to GIn/Ala would make the enzyme resemble the intermediate state. I need to read all the way until Figure 9 and the Discussion to be informed about the Phe-residue that flips between open/closed conformations.

We have added a new Figure (now Figure 2) together with some explanatory text in the introduction to make this clearer.

After reading the paper, it is still not clear to me whether GIn was tried, or whether other amino acids could have had the same effect. Perhaps it is not important since Ala apparently worked.

We have initially tried the GIn variant, but because this was less stable, we decided to work with the Ala variant instead. We have added this explanation to the end of the introduction.

In the Abstract, acyl-intermediate could more clearly be written as acyl-enzyme intermediate.

Done.

Results, Protein expression, and purification, TEV site would more precisely be termed TEV protease cleavage site.

Done.

Results, Crystallization (Figure 2) C164A/C161A is mixed non-consistently. I can only assume this is a mix-up with the group's other work with FabF. But it is very confusing to the reader.

This was indeed a mix-up with the number from FabF which has now been corrected (figure legend for what is now Figure 3).

Clearly out of scope to the paper, but it would have been interesting to know if iodine-phasing was tested.

We agree that this would be interesting, but we have not yet tested this. We might try it in the future.

It is unclear how the resolution cutoff was decided. I/sig(I) is still very high at the highest resolution shell. It would also have been useful if CC* or CC1/2 was presented in Table 2

The resolution was limited by the distance of the detector. Unfortunately, we had major problems with shipping crystals to the synchrotron this spring and lost two consecutive shipments due to shipping/custom problems. As the work presented in this paper is from a master thesis, there was no time to repeat the measurements, but it looks like the crystals might diffract even beyond $1.3 \AA$. We have added CC1/2 to Table 2 .

The very short Discussion-segment could have been merged with the Results-section. The discussion does raise the very interesting possibility of dual-target inhibitors that would presumably be of high interest with regards to the emergence of resistance. This could have been emphasized earlier as well.

We have renamed the Results section to Results and Discussion and the old Discussion section to Conclusions. The discussion about dual-target inhibitors for both, the malonyl- 
CoA binding site and the fatty acid channel (see comments from Klaus Reuter) has been added to what is now the Results and Discussion section.

Methods, The sonication amplitude appears to lack a unit, presumably \%. For the protein concentration determination, the extinction coefficient used should be given.

Done

Reference 21 appears to be a mistake.

Deleted.

We hope that we have answered to all issues that you have raised to your full satisfaction. If not, please let us know what else we should address.

Competing Interests: No competing interests were disclosed.

The benefits of publishing with F1000Research:

- Your article is published within days, with no editorial bias

- You can publish traditional articles, null/negative results, case reports, data notes and more

- The peer review process is transparent and collaborative

- Your article is indexed in PubMed after passing peer review

- Dedicated customer support at every stage

For pre-submission enquiries, contact research@f1000.com 